Article

\title{
Anti-Corrosion Characteristics of Electrodeposited Self-Doped Polyaniline Films on Mild Steel in Low Acidity
}

\author{
Jhen-Wei Wu ${ }^{1}$, Tzong-Liu Wang ${ }^{1}$, Wen-Churng $\operatorname{Lin}^{2}{ }^{\mathbb{D}}$, Hung-Yin Lin ${ }^{1}$, Mei-Hwa Lee ${ }^{3}$ and \\ Chien-Hsin Yang ${ }^{1, *}$ \\ 1 Department of Chemical and Materials Engineering, National University of Kaohsiung, Kaohsiung 81148, \\ Taiwan; haha9337@gmail.com (J.-W.W.); tlwang@nuk.edu.tw (T.-L.W.); linhy@nuk.edu.tw (H.-Y.L.) \\ 2 Department of Environmental Engineering, Kun Shan University, Tainan 71070, Taiwan; \\ linwc@mail.ksu.edu.tw \\ 3 Department of Materials Science and Engineering, I-Shou University, Kaohsiung 84001, Taiwan; \\ mhlee@isu.edu.tw \\ * Correspondence: yangch@nuk.edu.tw; Tel.: +886-7-591-9420
}

Received: 7 February 2018; Accepted: 19 April 2018; Published: 25 April 2018

\begin{abstract}
Without the addition of inorganic acids, 2,5-Diaminobenzenesulfonic acid (DABSA) molecules form an acid environment, and are then electrochemically copolymerized with AN monomers to generate a self-doped polyaniline (SPAN) film on mild steel substrates. These SPAN deposition films are employed to test the protection efficiency for mild steel in a corrosion environment of $\mathrm{HCl}$ and $\mathrm{NaCl}$, respectively. Electrochemical impedance spectroscopy (EIS) and polarization were used to determine the charge transfer resistance $\left(R_{\mathrm{ct}}\right)$ and corrosion current $\left(I_{\mathrm{corr}}\right)$, respectively. The above two parameters $R_{\mathrm{ct}}$ and $I_{\text {corr }}$ are combined to evaluate the protection efficiency of SPAN film on mild steel. Experimental results show that the SPAN thin film with the AN/DABSA ratio of 8.8 has the optimal corrosion resistance in $1 \mathrm{M} \mathrm{HCl}$ and $1 \mathrm{M} \mathrm{NaCl}$ aqueous solutions, respectively.
\end{abstract}

Keywords: mild steel; self-doped polyaniline; anti-corrosion; electrochemical impedance; polarization

\section{Introduction}

Corrosion occurs in consecutive oxidation reactions when metal is exposed to an erosive species, such as $\mathrm{O}_{2}$ and $\mathrm{H}^{+}$, resulting in the deterioration of metal. Corrosion causes material defects, holes, and even cracks. Therefore, anti-corrosion is a very common and important issue in material science. Various methods have been developed to resist corrosion, for example, chrome treatment of steel surfaces, pickling inhibitors, sacrificial zinc layers, etc. [1]. All of the above methods of corrosion protection are involved in one of the following ways: (i) in cathodic protection, an electron is donated to the steel from a sacrificial material; (ii) in anodic protection, an electron is withdrawn from the steel; or (iii) the steel surface is sealed off from corrosive attacks by a surface layer of another material. Cathodic protection of protective coating is widely used [2-4]. On the other hand, organic and polymeric coatings have been also employed to protect metals against corrosion. These coatings are employed to form a barrier between erosive species and metals, preventing metals from contacting these erosive species, reducing oxidation [5]. Because conductive polyaniline (PAN) coatings have been demonstrated to have better anticorrosion properties than other polymers, PAN-functionalized anticorrosion coatings have been extensively studied in the past decades [6-8]. Although the mechanism for enhanced corrosion protection has been attributed to an increase in the corrosion potential and the redox catalytic capability of PAN in the formation of a passive metal oxide layer [9-11], the precise mechanism of this corrosion control on metals via PAN films has yet to be fully clarified [12]. 
A galvanic interaction occurs between metal and PAN with the oxidized and conducting form of PAN, emeraldine salt (ES), which is usually formed by doping the reduced form of leucoemeraldine base (EB) with a doping acid. The galvanic interaction between PAN ES and the metal substrate leads to the polarization of the metal substrate to more positive potentials with the formation of PAN EB and the subsequent release of the dopant anion. The galvanic interaction forms a protective PAN-film-induced oxide layer generated via the cyclic reduction of PAN ES to PAN EB, with the subsequent re-oxidation of the latter species by $\mathrm{O}_{2}$, the formation of a passivating salt film at the PAN metal interface, and the suppression of $\mathrm{O}_{2}$ reduction as a result of a shift to more positive potentials. On an iron substrate, the nature of the dopant determines the characteristics of the PAN-induced layer, forming perhaps an oxide or a salt layer. Dopants would not assist in the formation of an oxide layer where there are defects.

There are various processes to synthesize polyaniline derivatives, such as chemical oxidation polymerization and electrochemical polymerization. There are several problems with the direct application of conducting polymer coatings. Their application for corrosion protection on active metals becomes difficult because conducting polymers are insoluble and non-fusible [5]; electrochemical polymerization is an alternative method to directly deposit polyaniline film on the surface of the metal piece. However, the electrochemical deposition of polyaniline is usually performed in a strong inorganic acidic solution, limiting its usefulness for metal protection. To avoid the erosive effect of the strong acid environment on the metal substrate, in this study we alternatively adopted self-doping aniline monomers in the electrochemical polymerization. Self-doped monomers will participate in electrochemical polymerization to deposit a self-doped polyaniline film on metal substrates. Sulfonate groups were incorporated into the polymer backbone of polyaniline, which altered the ion exchange properties of the polymer, giving rise to a self-doping effect. There are several advantages to the increased solubility of monomers in aqueous solution and the remaining conductivity in a wider $\mathrm{pH}$ range [13]. Furthermore, the product of self-doped polyaniline possesses chemical stability, thermal stability, etc. [13-17], greatly increases the applicability of self-doped polyaniline.

In this work, the self-doped polyaniline (SPAN) was co-deposited on a mild steel substrate using electrochemical polymerization in 2,5-diaminobenzenesulfonic acid (DABSA) and aniline (AN) co-monomers. Here, DABSA is designed to perform both roles of a di-functional (di-amono-)monomer and a doping acid. The acidity of DABSA is relatively low compared to inorganic acids, and it is desirable to reduce the occurrence of erosive reactions and to make polyaniline adhere better to the mild steel substrate during electropolymerization. An electrochemical impedance test and the polarization method were employed to evaluate the substantial anti-corrosion effect of the SPAN films on mild steel in $1 \mathrm{M} \mathrm{HCl}$ and $1 \mathrm{M} \mathrm{NaCl}$ solutions, respectively.

\section{Materials and Methods}

\subsection{Materials}

Reagent grade aniline (Merck, Kenilworth, NJ, USA) was doubly distilled and the resulting colorless liquid kept under nitrogen in darkness at $5{ }^{\circ} \mathrm{C}$. Reagent grade 2,5-diaminobenzenesulfonic acid (Aldrich, Milwaukee, WI, USA), hydrochloride (Scharlau, Mas d'en Cisa, Spain), and sodium chloride (Showa, Kyushu, Japan) were used as received. Mild steel (model SPCC, China Steel Corporation, composition (wt \%) of mild steel: $\mathrm{C} \leq 0.15, \mathrm{Mn} \leq 0.60, \mathrm{P} \leq 0.10$, and $\mathrm{S} \leq 0.05$ ) was kindly provided by the China Steel Corporation (Kaohsiung, Taiwan). Indium-tinoxide (ITO)-coated glass $(8 \Omega / \square)$ was purchased from Merck (Kenilworth, NJ, USA).

\subsection{Preparation of Test Samples}

Mild steel $\left(4 \times 1.5 \mathrm{~cm}^{2}\right)$ was used as the corrosion-test substrate. The surface rust of the mild steel was sequentially removed using abrasive papers (Models 400, 600, and 800). The impurity on the surface was thoroughly washed using acetone, alcohol and distilled water. The surface of 
indium-tinoxide (ITO)-coated glass was thoroughly washed using acetone, alcohol and distilled water. A test piece of $1 \mathrm{~cm}^{2}$ area was employed to perform the subsequent experiments.

\subsection{Electrochemical Polymerization of SPAN on Mild Steel}

Electrochemical experiments were performed using an electrochemical analyzer (PGSTAT 30, Metrohm Autolab, Utrecht, The Netherlands). All measurements were carried out at room temperature with a conventional three-electrode configuration consisting of a pre-treated mild steel piece, a platinum plate auxiliary electrode, and an aqueous $\mathrm{Ag} / \mathrm{AgCl}$ reference electrode. SPAN was deposited electrochemically at $25{ }^{\circ} \mathrm{C}$ by cyclic voltammetry on a mild steel electrode in aqueous solutions $(70 \mathrm{~mL})$ containing constant molarities $(0.2 \mathrm{M})$ of $-\mathrm{NH}_{2}$ with three AN/DABSA molar ratios of 3.4, 8.8, and 10. The potential range was swept from -200 to $800 \mathrm{mV}$ at a scan rate of $25 \mathrm{mV} / \mathrm{s}$. To compare the different composition polymers, the reaction times were chosen to control the approximately same amount of polymer deposited on the electrode corresponding to the respective comonomer feed ratio. After completing the electropolymerization, the SPAN-deposited mild steel piece was washed using distilled water to remove residual monomers and then placed in the oven to dry at $60^{\circ} \mathrm{C}$ for $12 \mathrm{~h}$.

\subsection{Materials Analysis}

The microstructure of the sample was investigated by scanning electron microscopy (SEM, S-4800, Hitachi, Tokyo, Japan). Infrared spectra were recorded on an FTIR spectrometer (Agilent Technologies, Cary 630, Santa Clara, CA, USA) to check the functional groups on polymer films.

\subsection{Electrochemical Measurements}

Two methods of electrochemical impedance and polarization were employed to study the corrosion protection in the test solutions of $1 \mathrm{M} \mathrm{HCl}$ and $1 \mathrm{M} \mathrm{NaCl}$, respectively. After electropolymerization, the test piece of SPAN-deposited mild steel was immersed in the test solution for $15 \mathrm{~min}$ to perform the above electrochemical measurements. AC impedance was measured in the frequency range of $10 \mathrm{kHz}$ and $10 \mathrm{mHz}$ with an $\mathrm{AC}$ amplitude of $10 \mathrm{mV}$. The data on the $\mathrm{AC}$ impedance were plotted in a Nyquist plot including the real and the imaginary parts. The charge transfer resistance $\left(R_{\mathrm{ct}}\right)$ was obtained from the semicircular diameter in the real part of the Nyquist plot. The $R_{\mathrm{ct}}$ value was substituted into the following equation to obtain the protection efficiency [2]:

$$
\text { Protection efficiency }(\%)=\left\{\left[1 / R_{\mathrm{ct}}-1 / R_{\mathrm{ct}(\mathrm{c})}\right] /\left(1 / R_{\mathrm{ct}}\right)\right\} \times 100
$$

where $R_{\mathrm{ct}}$ and $R_{\mathrm{ct}(\mathrm{c})}$ are the blank test and the SPAN-deposited test pieces, respectively.

The polarization measurement was within the potential range of $-200 \sim+200 \mathrm{mV}$ (open-circuit potential, OCP) at a scanning rate of $1 \mathrm{mV} / \mathrm{s}$. The polarization curve consists of anodic and cathodic polarization curves. The intersection of these two curves corresponds to the corrosion potential. The corrosion current can be evaluated by the method of Tafel extrapolation. In the linear regions of the cathodic-anodic Tafel area, corresponding to the corrosion potential $\pm 70 \sim 120 \mathrm{mV}$, the slopes of the two linear regions are extended to the intersection point that corresponds to the corrosion current [18]. The corrosion rate (C.R.) can be obtained by substituting the corrosion current into the following equation [2]:

$$
\text { C.R. }(\mathrm{mm} / \text { year })=\left[3.27 \times 10^{-3} \times I_{\text {corr }} \times M\right] /(n \rho)
$$

where $I_{\text {corr }}$ is the corrosion current $\left(\mu \mathrm{A} / \mathrm{cm}^{2}\right), M$ is the molecular weight of the metal, $\rho$ is the metal density $\left(\mathrm{g} / \mathrm{cm}^{3}\right)$, and $n$ is the metal valence. 


\section{Results and Discussion}

\subsection{Electrodeposition of SPAN}

Figure 1 shows the cyclic voltammogramms (CVs) of DABSA-AN electropolymerization on an ITO electrode in $1 \mathrm{M} \mathrm{HCl}$. In this acidic solution, the current gradually increases with the increasing cycling number of the electropolymerization (refer to Figure 1a-c), due to the increase of the conductive polymer. The electropolymerization of polyaniline requires attachment and chain extension between the active intermediates of the diradical dications [19] generated from the monomers and the propagating oligomer chains, as shown in Scheme 1. The diradical dication is an energetic electrophile, thus polymerization is generally performed in the presence of a strong acid (e.g., $1 \mathrm{M}$ $\mathrm{HCl})$ to deposit polyaniline on the electrode. Since DABSA has two amino groups $\left(-\mathrm{NH}_{2}\right)$ in the ortho and para position on the benzene ring, DABSA molecules more readily generate diradical dications (the bipolaronic form of pernigraniline) than AN molecules, which corresponds to peak B $(\sim 800 \mathrm{mV})$. This species is an energetic electrophile, extracting an electron from aniline and becoming a radical cation, which bonds with another radical, indicating by the increase in the intensity of peak A at $\sim 250 \mathrm{mV}$ (referring to the 15th cycle in Figure 1a), that these radical cations undergo bonding to form the polymer deposited on the ITO anode surface. A comparison of Figure 1a-c reveals that the intensity of peak A decreases with increasing the DABSA content and gives rise to more nucleation sites (quinonediimines), increasing the possibility of attachment of these DABSA-generated nucleation sites. This kind of DABSA attachment leads to a failure in the chain extension, resulting in the suppression of the intensity of peak A (refer to Figure $1 b, c)$. The electropolymerization of SPAN was performed on an ITO substrate in $1 \mathrm{M} \mathrm{HCl}$, consisting of AN and DABSA comonomers using cyclic voltammetry, demonstrating that ITO can resist this strong acid environment and successfully deposit SPAN films on it. In contrast, mild steel was used to replace the ITO substrate, because the strong acid environment will directly corrode mild steel substrates and fail to deposit SPAN films on this steel. Therefore, we tried to run the electropolymerization of DABSA and AN on an ITO electrode without adding inorganic acid $(\mathrm{HCl})$. The CVs of DABSA-AN electropolymerization on an ITO electrode obtained in weak acidic solution are shown in Figure 2, revealing that the current tendency is opposite to the situation illustrated in Figure 1. This result indicates that the monomers of DABSA and AN adsorb on the ITO surface by driving electrochemical potential in the first sweep, which generate diradical dications that are difficult to keep energetic in a weak acidic environment, leading to a failure of the chain extension in later cycling sweeps. Consequently, these deposits are speculated to exist in the form of oligomers with relatively low electrical conductivity, since these deposits possess low conductivity and contain lone-pair electrons on nitrogen atoms in the SPAN oligomers, which are suitable for the anti-corrosion coating of metals. However, a mild steel sheet cannot be used in a strong acid aqueous solution as the electrode for depositing polyaniline, because this steel sheet is easily corroded so that polyaniline cannot be successfully deposited on it. Therefore, we mixed the sulfonate-containing DABSA with AN as the comonomer to make a weak acidic aqueous solution without adding inorganic acid. This weak acidic comonomer solution did not corrode the steel sheet, and was then subjected to SPAN electrodeposition on the mild steel electrode. The CVs of DABSA-AN electropolymerization on a mild steel electrode obtained in weak acidic solution are shown in Figure 3, revealing that the current tendency is opposite to the situation illustrated in Figure 1, and the electrodeposition behavior is almost the same as that on an ITO electrode, as seen in Figure 2. In the present study, it is presumed that these SPAN deposits on mild steel substrates can be further employed to test anti-corrosion in $\mathrm{HCl}$ and $\mathrm{NaCl}$. 
(a)

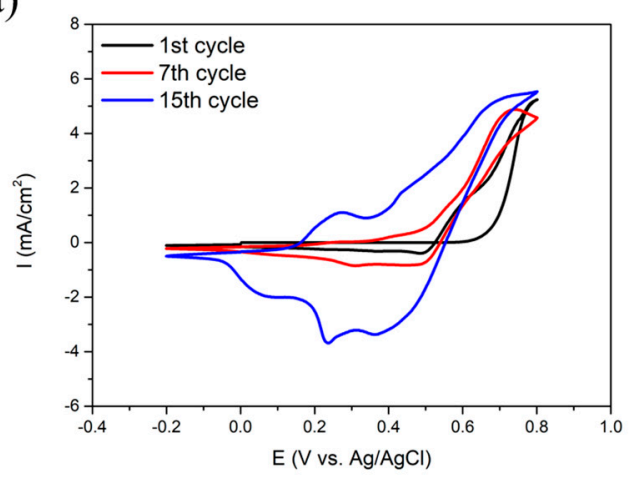

(b)

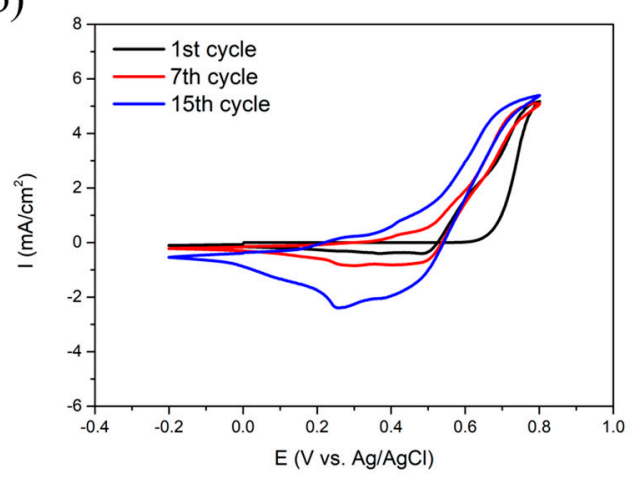

(c)

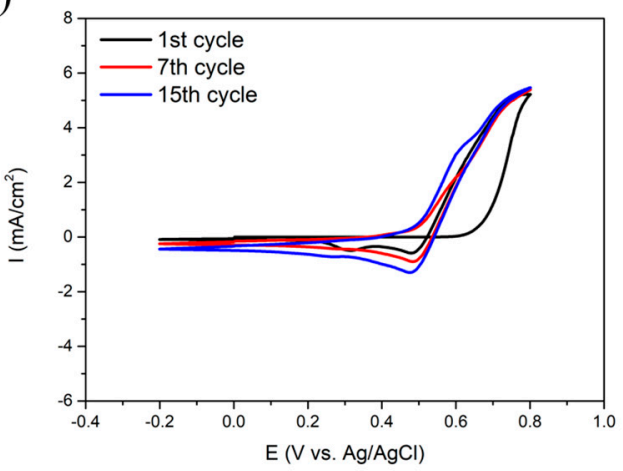

Figure 1. Cyclic voltammograms of SPAN deposited on an ITO electrode using electropolymerization in $1 \mathrm{M} \mathrm{HCl}$ with AN/DABSA mole ratio of (a) $10(\mathrm{pH}=-0.17)$, (b) $8.8(\mathrm{pH}=-0.19)$, and (c) 3.4 $(\mathrm{pH}=-0.21)$ at a scan rate of $25 \mathrm{mV} / \mathrm{s}$.

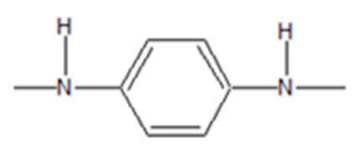

Amine (leucoemeraldine)

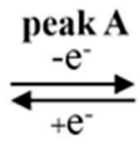

Radical cation (polaronic emeraldine)

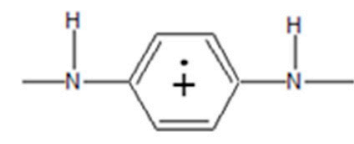

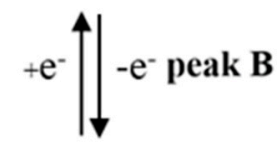

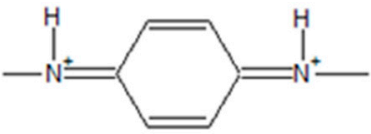

Protonated quinonediimine 
(a)

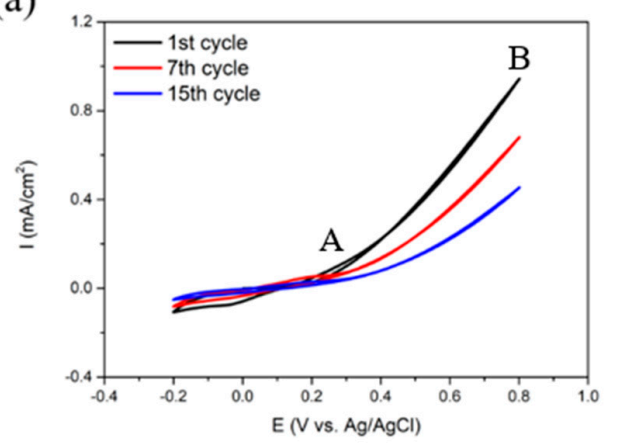

(b)

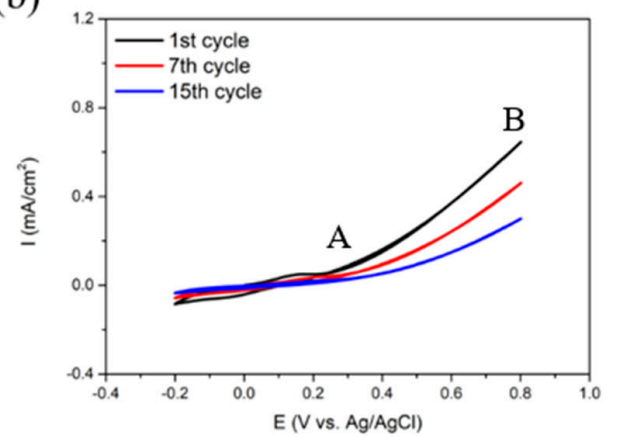

(c)

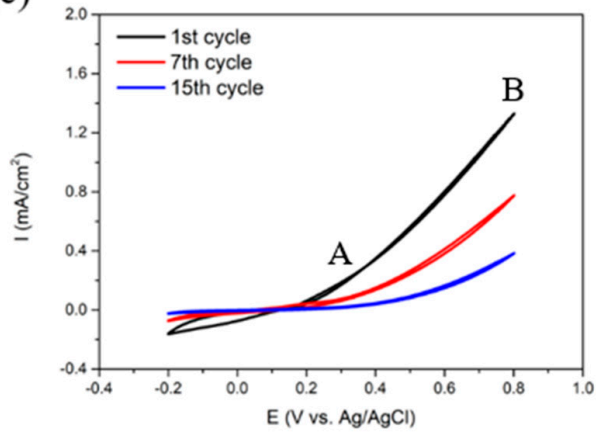

Figure 2. Cyclic voltammograms of SPAN deposited on an ITO electrode using electropolymerization with AN/DABSA mole ratio of (a) $10(\mathrm{pH}=6.24),(\mathbf{b}) 8.8(\mathrm{pH}=6.12)$, and (c) $3.4(\mathrm{pH}=5.86)$ at a scan rate of $25 \mathrm{mV} / \mathrm{s}$.

(a)

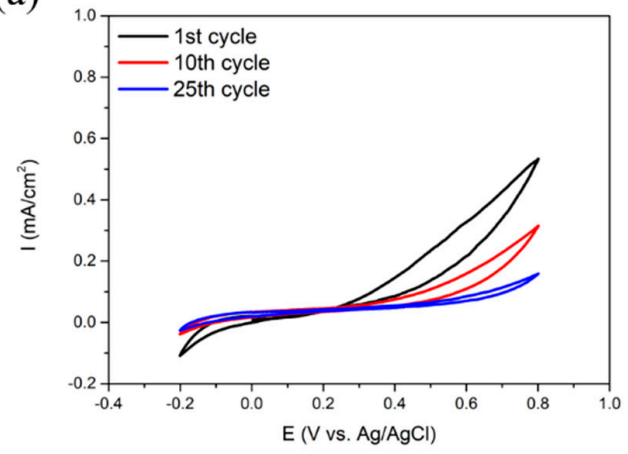

(b)

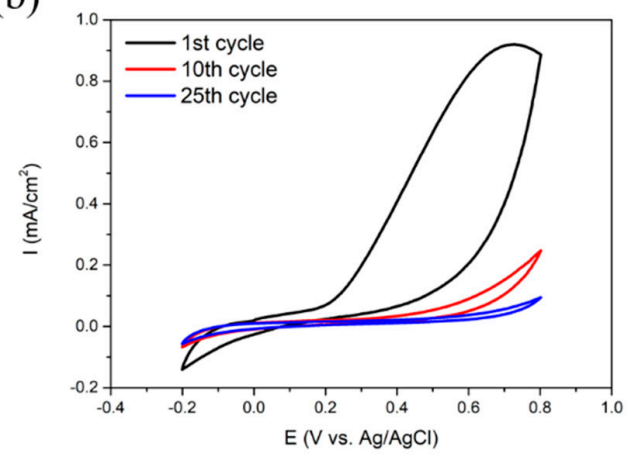

(c)

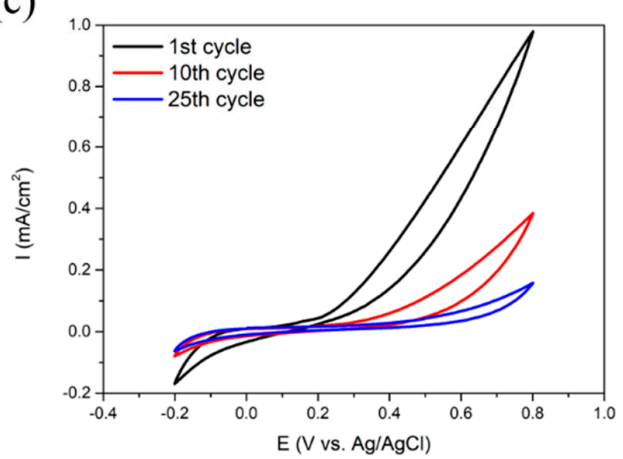

Figure 3. Cyclic voltammograms of SPAN deposited on a mild steel electrode using electropolymerization with AN/DABSA mole ratio of (a) $10(\mathrm{pH}=6.24),(\mathbf{b}) 8.8(\mathrm{pH}=6.12)$, and (c) $3.4(\mathrm{pH}=5.86)$ at a scan rate of $25 \mathrm{mV} / \mathrm{s}$. 


\subsection{Material Analysis}

\subsubsection{Morphology of SPAN Deposits}

Figure 4 shows photos of mild steel surfaces before and after abrasion using sandpaper, as well as SPAN deposited on the working area for the anti-corrosion test, demonstrating that there was significant rust on the surface of the mild steel before abrasion using sandpaper, and that a bright surface can be observed after abrasion. The pale purple SPAN-deposit on the surface of mild steel from the CV electropolymerization should also be noted. SEM images of the mild steel and SPAN deposited on mild steel substrates are shown in Figure 5. It is obvious that the surface of the mild steel substrate has a flat appearance (Figure 5a). In Figure 5b-d, SPAN can be successfully deposited on the surface of the mild steel substrate, demonstrating a substantial deposit. Figure 5e,f show that the SPAN films are still attached to the surface after the $\mathrm{AC}$ impedance tests in the $1 \mathrm{M} \mathrm{HCl}$ and $1 \mathrm{M} \mathrm{NaCl}$ solutions, respectively. To study the anti-corrosion characteristics of these SPAN films, each film deposition was swept about fifteen cycles using cyclic voltammetry to control a thickness of $100 \pm 10$ nm (refer to Figure 6).

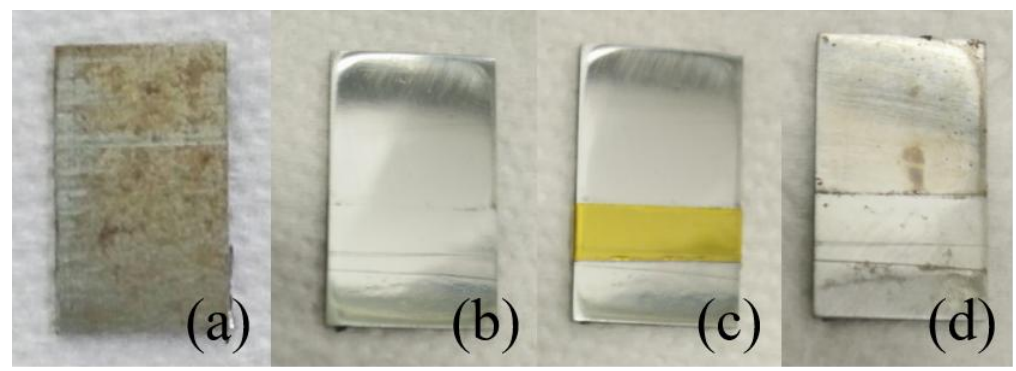

Figure 4. Photos of mild steel rust surface (a) before abrasion using sandpaper and (b) after abrasion; (c) $1 \times 1 \mathrm{~cm}^{2}$ working area after abrasion; and (d) SPAN deposited on the working area for anti-corrosion test. Here, SPAN deposited with an AN/DABSA ratio of 8.8.

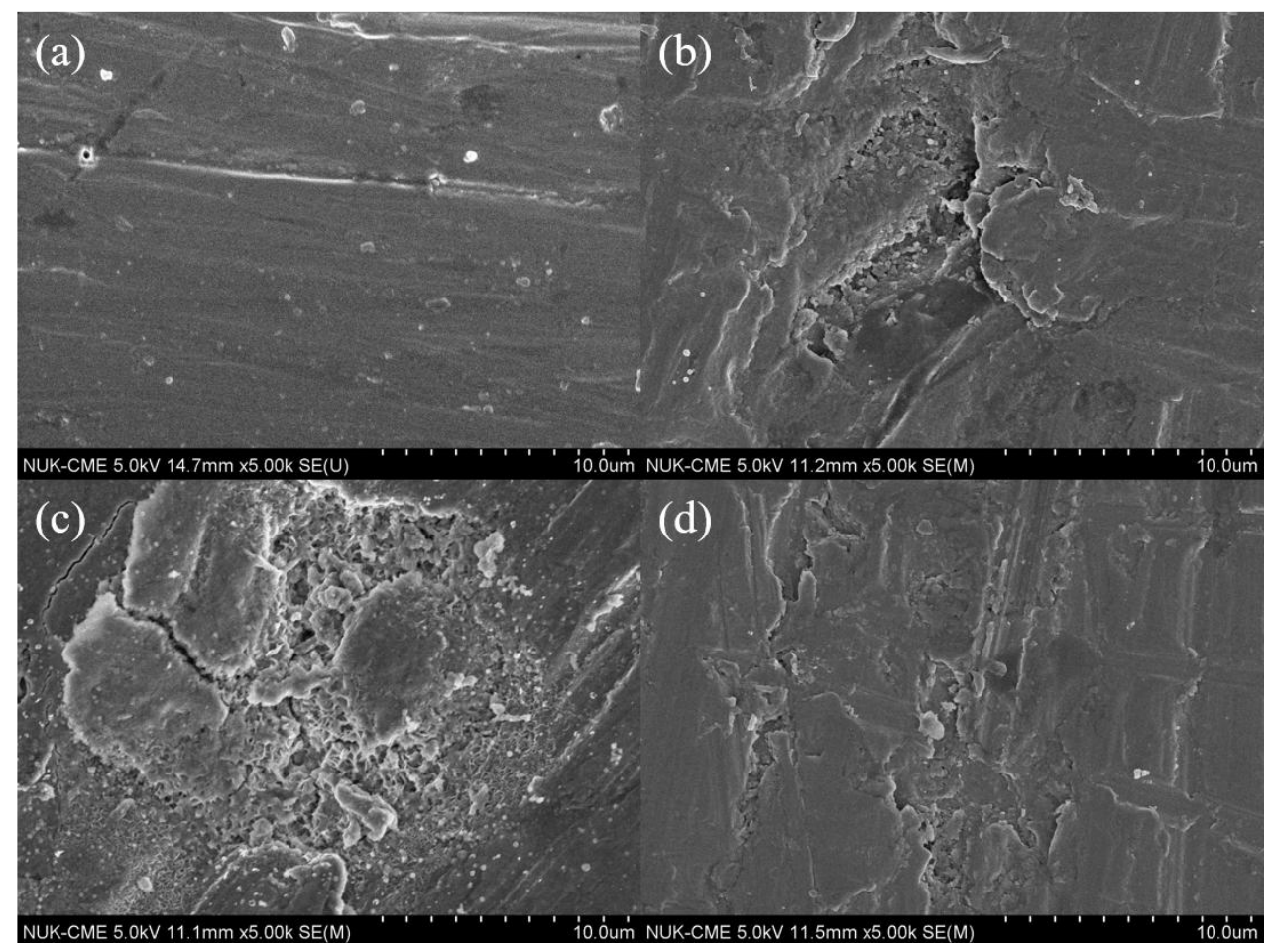

Figure 5. Cont. 


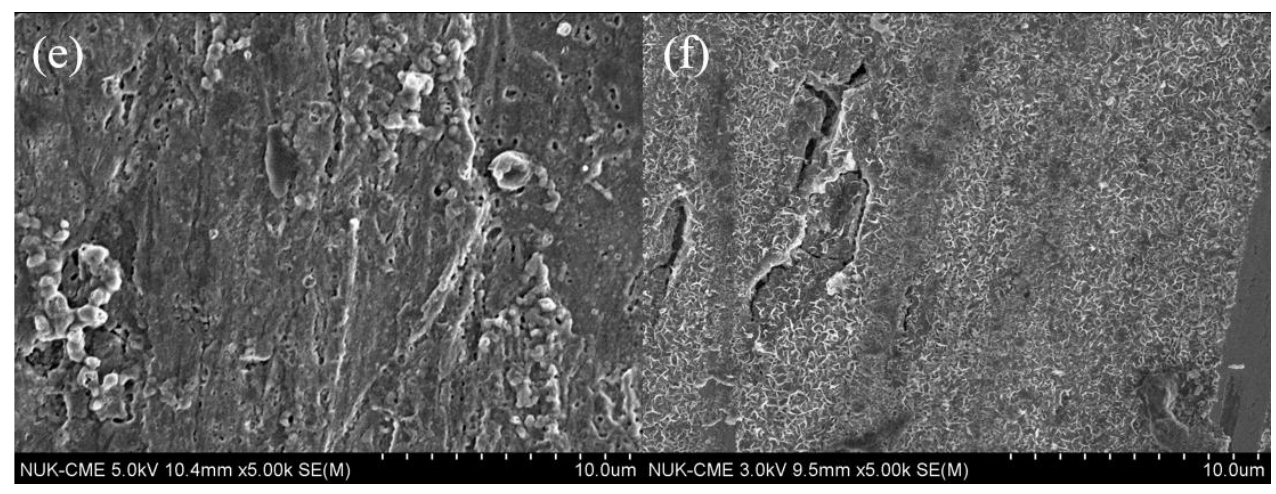

Figure 5. SEM images of SPCC mild steel (a); SPAN deposited on mild steels with an AN/DABSA ratio of $10(\mathbf{b}), 8.8(\mathbf{c})$, and $3.3(\mathbf{d})$; SPAN-deposited with AN/DABSA ratio of 8.8 on mild steel after an AC impedance test in $1 \mathrm{M} \mathrm{HCl}(\mathbf{e})$, and in $1 \mathrm{M} \mathrm{NaCl}(\mathbf{f})$.

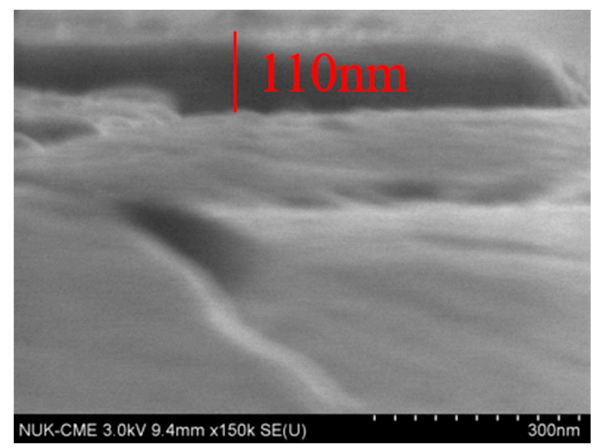

Figure 6. SEM cross-sectional image of SPAN deposited on a mild steel substrate with an AN/DABSA ratio of 8.8 swept fifteen cycles using cyclic voltammetry.

\subsubsection{FTIR Spectra}

Figure 7 shows FTIR spectra of SPAN electrodeposited on mild steel with an AN/DABSA ratio of 8.8, revealing that the out-of-plane and in-plane $\mathrm{C}-\mathrm{H}$ bonds appear at peaks of 837 and $1190 \mathrm{~cm}^{-1}$ [20], respectively, and that the vibration of the benzene rings and quinone rings are located at the peaks of 1515 and $1626 \mathrm{~cm}^{-1}$ [20], respectively; whereas the peak of $3363 \mathrm{~cm}^{-1}$ corresponds to $\mathrm{N}-\mathrm{H}$ stretching [20]. In this work, self-doped polyaniline SPAN) was electrodeposited on a mild steel substrate, and as a result the sulfonate on DABSA was involved in the spectra, which was centered at $1041 \mathrm{~cm}^{-1}$ corresponding to the $\mathrm{S}=\mathrm{O}$ bond [21].

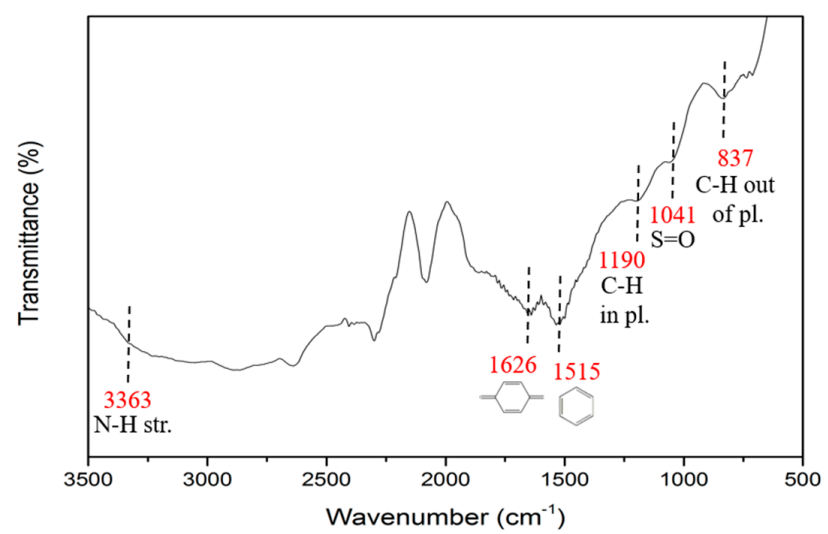

Figure 7. FTIR spectra of SPAN electrodeposited on mild steel with an AN/DABSA ratio of 8.8. 


\subsection{Characterization of the Anticorrosion Properties of the Deposited Polymer Layers}

\subsubsection{Electrochemical Measurements}

\section{Corrosion Test in $\mathrm{HCl}$}

Electrochemical impedance is a common method used to study the mechanism of corrosion. In corrosion tests, the Nyquist plot measured by electrochemical impedance shows a semicircle in the testing media [22], corresponding to the equivalent circuit, as shown in Figure 8, in which $R_{\mathrm{S}}$ represents the electrolyte resistance, $R_{\mathrm{ct}}$ is the charge transfer resistance between the electrolyte and electrode, and CPE stands for the double layer capacitance on the electrode surface [23-25].

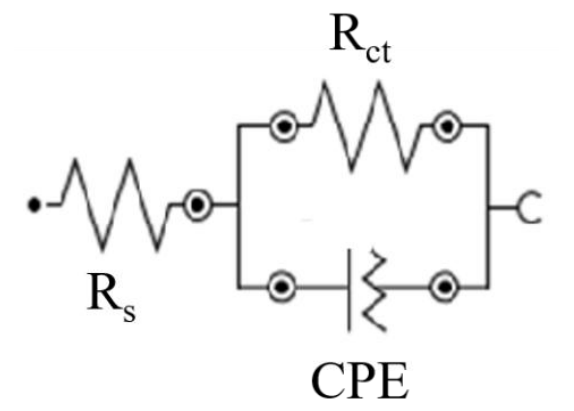

Figure 8. The equivalent circuit of corrosion tests.

The Nyquist plots obtained by the AC impedance test in $1 \mathrm{M} \mathrm{HCl}$ solution are shown in Figure 9, revealing that the charge transfer resistance $\left(R_{\mathrm{ct}}\right)$ of SPAN deposited on mild steels is several hundred times higher than that of the blank sample (mild steel). The highest charge transfer resistance exists on the SPAN film with AN/DABSA of 8.8. The charge transfer resistance and the protection efficiency are listed in Table 1. An examination of Table 1 reveals that the protection efficiency (PE) of electrodeposited SPAN films is more than $95 \%$ in $1 \mathrm{M} \mathrm{HCl}$ solution. In addition, the best efficiency of $97.4 \%$ corresponds to the SPAN film with AN/DABSA ratio of 8.8. One possible explanation for this result arises from the composition of the deposited film, which has a relatively dense structure covered in steel. At the highest AN/DABSA ratio of 10, the corresponding SPAN film was suddenly extra-doped by $1 \mathrm{M} \mathrm{HCl}$ to significantly increase the electrical conductivity (i.e., the lowest $R_{\mathrm{ct}}$ value in Table 1), resulting in lower protection efficiency.

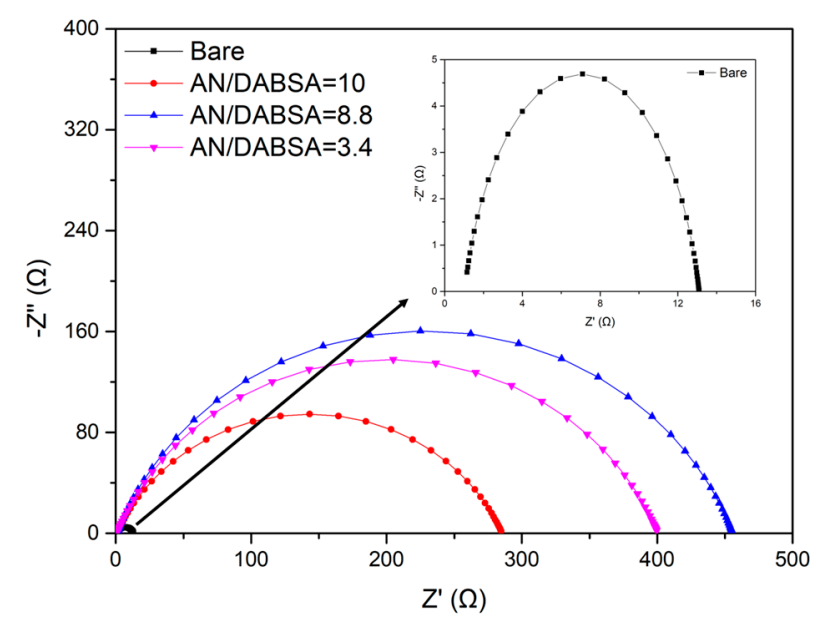

Figure 9. Nyquist plots of bare and SPAN-deposited mild steels with different AN/DABSA ratios in $1 \mathrm{M} \mathrm{HCl}$. 
Table 1. Charge transfer resistance $\left(R_{\mathrm{ct}}\right)$ derived from EIS measurements (Figure 9$)$ and the protection efficiency (PE) of bare and SPAN-deposited mild steels with different AN/DABSA ratios in $1 \mathrm{M} \mathrm{HCl}$.

\begin{tabular}{ccc}
\hline Sample & $\boldsymbol{R}_{\mathbf{c t}}\left(\boldsymbol{\Omega} \mathbf{~ c m}^{\mathbf{2}}\right)$ & PE (\%) \\
\hline Bare & $12.0(0.16)$ & - \\
AN $/$ DABSA $=10$ & $283.4(1.51)$ & $95.8(0.51)$ \\
AN/DABSA $=8.8$ & $453.5(3.24)$ & $97.4(0.70)$ \\
AN/DABSA $=3.4$ & $398.0(2.16)$ & $97.0(0.53)$ \\
\hline \multicolumn{3}{l}{ Note: The data in parentheses represent the standard deviation. }
\end{tabular}

Figure 10 demonstrates the polarization curve obtained by polarization in $1 \mathrm{M} \mathrm{HCl}$ solution. The electrochemical data obtained using the Tafel extrapolation method are listed in Table 2, revealing that there is no significant difference in the corrosion potential ( $\left.E_{\text {corr }}\right)$. On the three SPAN films with different ratios of AN/DABSA, the nitrogen atoms on the polyaniline molecular chains can be completely doped by $\mathrm{H}^{+}$in $1 \mathrm{M} \mathrm{HCl}$ (strong acid), so that the $E_{\text {corr }}$ values were almost the same. It should also be noted that the corrosion current $\left(I_{\text {corr }}\right)$ of the test piece with the SPAN-deposited film was much lower corresponding to the increase in polarization resistance $\left(R_{\mathrm{p}}\right)$. It should further be noted that the corrosion rate (C.R.) of the SPAN-deposited films was from 12 to 14 times lower than that of the blank test piece (mild steel).

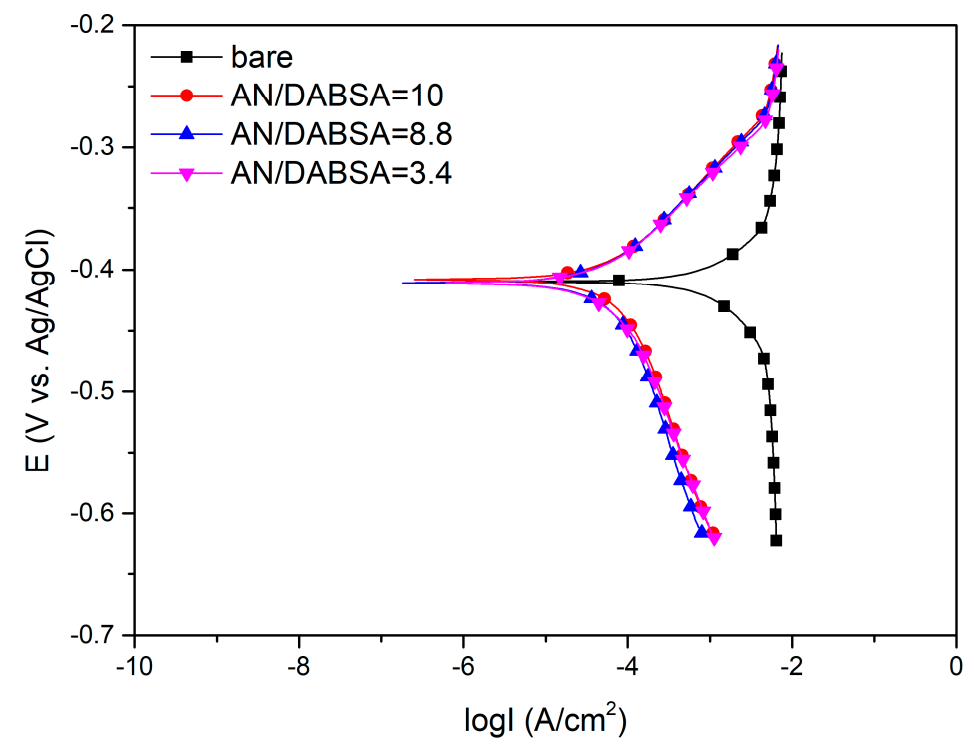

Figure 10. Polarization curves of bare and SPAN-deposited mild steels with different AN/DABSA ratios in $1 \mathrm{M} \mathrm{HCl}$.

Table 2. Electrochemical parameters obtained using polarization on bare and SPAN-deposited mild steels with different AN/DABSA ratios in $1 \mathrm{M} \mathrm{HCl}$.

\begin{tabular}{ccccc}
\hline Sample & $\boldsymbol{E}_{\text {corr }}(\mathbf{m V}$ vs. $\mathbf{A g} / \mathbf{A g C l})$ & $\boldsymbol{I}_{\text {corr }}(\boldsymbol{\mu} \mathbf{A})$ & $\boldsymbol{R}_{\mathbf{p}}\left(\boldsymbol{\Omega} \mathbf{~ c m}^{\mathbf{2}}\right)$ & C.R. $\left(\mathbf{m m ~ y e a r}^{-\mathbf{1}}\right)$ \\
\hline Bare & $-410(4.14)$ & $953.0(9.62)$ & $16.6(0.17)$ & $11.11(0.11)$ \\
AN/DABSA $=10$ & $-408(6.16)$ & $78.8(1.19)$ & $288.0(4.34)$ & $0.92(0.01)$ \\
AN/DABSA $=8.8$ & $-411(5.59)$ & $66.9(0.91)$ & $331.2(4.50)$ & $0.78(0.01)$ \\
AN/DABSA $=3.4$ & $-411(2.68)$ & $69.0(0.45)$ & $300.5(1.96)$ & $0.80(0.05)$ \\
\hline
\end{tabular}

Corrosion is generally caused by an electrochemical inhomogeneity in a metal or its environment. In contact with an electrolyte, corrosion happens in metal areas with higher free energy (or higher potential), namely anodes, and those of lower free energy (or lower potential), namely cathodes, 
thereby generating a corrosion cell. Metal ions are produced at the anode and dissolve into the electrolyte. The electrons pass through the metal to the adjacent cathode areas that have reacted with the environment. Electrons flow from the anode to the cathode and the related charge transfers through the electrolyte from the cathode to the anode creating the corrosion current. The corrosion rate is, therefore, related to the corrosion current. The process of corrosion can be represented by the following reactions [7]:

Reactions of carbon steel in acid solutions with dissolved oxygen $[7,26]$ :

$$
\begin{gathered}
\text { Anode (oxidation): } \mathrm{Fe} \rightarrow \mathrm{Fe}^{2+}+2 \mathrm{e}^{-} \\
\text {Cathode (reduction): } \mathrm{O}_{2}+4 \mathrm{H}^{+}+4 \mathrm{e}^{-} \rightarrow 2 \mathrm{H}_{2} \mathrm{O}
\end{gathered}
$$

There are two steps in the oxidative rusting process of carbon steel in dissolved oxygen solution [7]:

$$
\begin{gathered}
\mathrm{Fe}+\frac{1}{2} \mathrm{O}_{2}+\mathrm{H}_{2} \mathrm{O} \rightarrow \mathrm{Fe}^{2+}+2 \mathrm{OH}^{-} \rightarrow \mathrm{Fe}(\mathrm{OH})_{2} \\
2 \mathrm{Fe}(\mathrm{OH})_{2}+\frac{1}{2} \mathrm{O}_{2}+\mathrm{H}_{2} \mathrm{O} \rightarrow 2 \mathrm{Fe}(\mathrm{OH})_{3}
\end{gathered}
$$

Ferric hydroxide, $\mathrm{Fe}(\mathrm{OH})_{3}$, is known normally as red rust and forms in the initial stages of corrosion, gradually changing to ferric oxy-hydroxide, $\mathrm{FeOOH}$. $\mathrm{Fe}(\mathrm{OH})_{3}$ further dehydrates to become ferric oxide:

$$
2 \mathrm{Fe}(\mathrm{OH})_{3} \rightarrow \mathrm{Fe}_{2} \mathrm{O}_{3}+3 \mathrm{H}_{2} \mathrm{O}
$$

SPAN coating can work as a barrier against the diffusion of the electrolyte $(\mathrm{HCl})$ and aggressive ions $\left(\mathrm{Cl}^{-}\right)$, protecting the substrate metal. In addition, the oxidation of metal can give rise to the reduction of SPAN (ES) to SPAN (EB) in the SPAN deposit film, leading to the anodic protection of a passive, protective $\mathrm{FeOOH}$ layer $[27,28]$. Chloride ions easily pass through the protective layer and reach the metal surface, thus accelerating the dissolution reaction. In the acidic solution, chloride ions would be inhibited to slow down the dissolution and corrosion of mild steel [7]. There exists a galvanic coupling between the metal and the SPAN (Scheme 2) when a scratch occurs on the SPAN-coated metal. The cathodic reaction involves the reduction of the SPAN associated with the prominent activation of the self-doping anions $\left(-\mathrm{SO}_{3}{ }^{-}\right)$, whereas the anodic reaction involves the oxidation of the metal. On the other hand, the $\mathrm{O}_{2}$ reduction occurs simultaneously on both the SPAN layer and metal surface, leading to the SPAN reoxidation and the production of $\mathrm{OH}^{-}$, respectively. A self-healing process might be initiated, depending on the nature of the metal and doping anions. In general, metal oxide formation is initiated by SPAN coatings, leading to the formation of a protective layer of metal oxides on the metal surface, preventing corrosion (anodic protection mechanism [7,29]), or the self-doping anions $\left(-\mathrm{SO}_{3}{ }^{-}\right)$act as inhibitors (controlled inhibitor release mechanism $\left.[7,30,31]\right)$. It seems that the sulfonate groups in SPAN act as inner doping anions, excluding an anion exchange process between the SPAN film and the chloride-containing solution. Several investigations have also shown this effect in the case of the polyaniline layer [30-33]. From Table 1, we can see that the anti-corrosion ability of the SPAN film with an AN/DABSA ratio of 10 is lower than that of the SPAN film with an AN/DABSA ratio of 8.8, because the content of the sulfonate anion in the former film is lower than that in the latter film. The low content of sulfonate anions is not conducive to preventing the $\mathrm{Cl}^{-}$anion from attacking the metal substrate through the deposited polymer layer, resulting in the corrosion of the metal substrate. Although the highest content of sulfonate anion is in SPAN film with an AN/DABSA ratio of 3.4, its hydrophilicity is also the highest, which offsets the effect of the high sulfonate anion blocking the attack of the $\mathrm{Cl}^{-}$anion on the metal substrate. As a consequence, we infer that the polymer film has the optimal AN/DABSA ratio for anti-corrosion. An AN/DABSA ratio of 8.8 is optimal. 


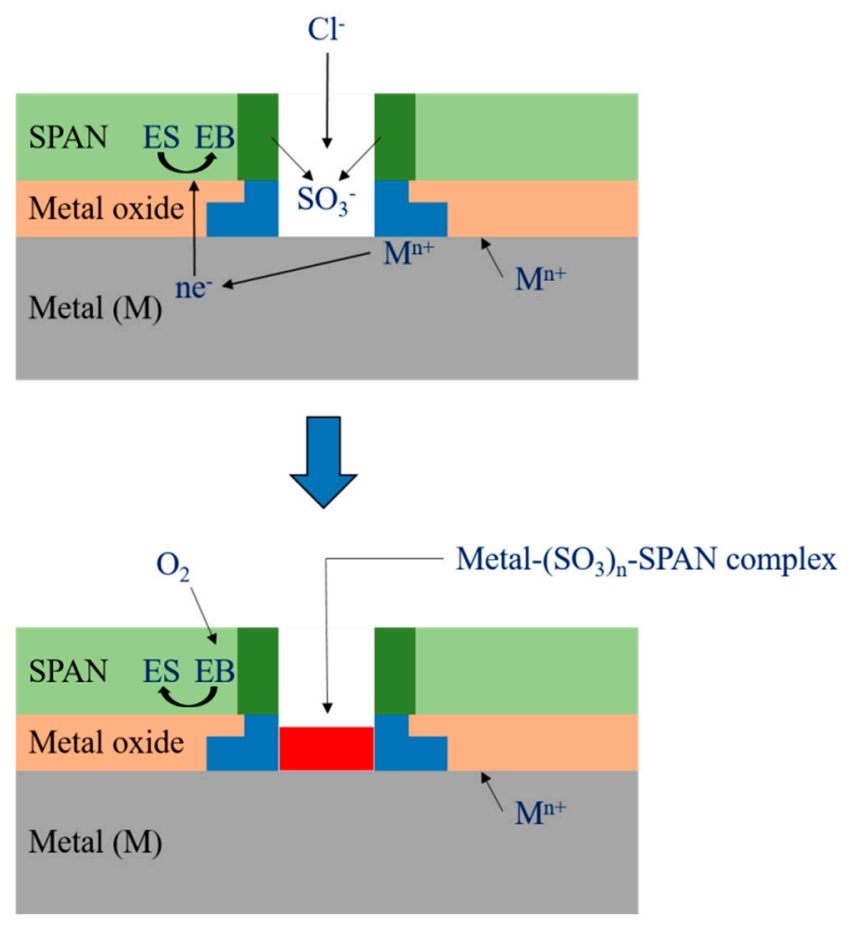

Scheme 2. Controlled inhibitor release mechanism for a metal (M)-coated SPAN layer self-doped with an anion, $\mathrm{SO}_{3}{ }^{-}$, which acts as a corrosion inhibitor.

\section{Corrosion Test in $\mathrm{NaCl}$}

Figure 11 shows Nyquist plots obtained by an $\mathrm{AC}$ impedance test in $1 \mathrm{M} \mathrm{HCl}$ solution, revealing that the charge transfer resistance $\left(R_{\mathrm{ct}}\right)$ of SPAN deposited on mild steels is several times higher than that of the blank sample (mild steel). The highest charge transfer resistance still exists on the SPAN film with an AN/DABSA of 8.8. The charge transfer resistance and the protection efficiency are listed in Table 3. It is obvious that the protection efficiency (PE) of electrodeposited SPAN films ranges from $50 \%$ to $83 \%$ in $1 \mathrm{M} \mathrm{NaCl}$ solution. In addition, the best efficiency of $82.9 \%$ corresponds to SPAN film with an AN/DABSA ratio of 8.8. This trend is consistent with that for $1 \mathrm{M} \mathrm{HCl}$ solution.

Figure 12 demonstrates the polarization curve obtained by polarization in $1 \mathrm{M} \mathrm{NaCl}$ solution. The electrochemical data obtained using the Tafel extrapolation method are listed in Table 4, revealing that there is significant increase in the corrosion potential $\left(E_{\text {corr }}\right)$ on SPAN-deposited samples, as compared to bare mild steel. The $E_{\text {corr }}$ values of the three SPAN films were different in $1 \mathrm{M} \mathrm{NaCl}$. In this case, $\mathrm{Na}^{+}$ions could not completely dope the nitrogen atoms on the polyaniline molecular chains in the obtained SPAN films, because the radius of $\mathrm{Na}^{+}$ion $(149 \mathrm{pm})$ was much larger than that of $\mathrm{H}^{+}(10 \mathrm{pm})$, leading to the difficult diffusion of $\mathrm{Na}^{+}$ions into the SPAN films to dope the nitrogen atoms of the polymer chains. As a result, the three films retained the doping degree of the original sulfonate on the DABSA molecules in the SPAN chains. The doping degree of the SPAN films with AN/DABSA ratio of 8.8 was greater than that with a ratio of 10, corresponding to the higher

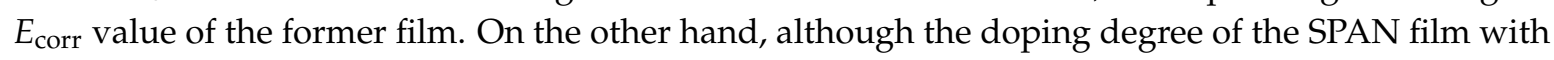
an AN/DABSA ratio of 3.4 was theoretically the highest, its solubility in water was also relatively high, resulting in an $E_{\text {corr }}$ value corresponding to that of SPAN film with an AN/DABSA ratio of 10. This indicates that the anti-corrosion ability increases in this SPAN-deposited mild steel, whereas the highest corrosion potential of $-523 \mathrm{mV}$ was achieved on the SPAN film with an AN/DABSA ratio of 8.8. The corrosion current $\left(I_{\text {corr }}\right)$ of the test piece with the SPAN-deposited film was lower, corresponding to the increase of polarization resistance $\left(R_{\mathrm{p}}\right)$. In addition, the corrosion rate (C.R.) of the SPAN-deposited films was two times lower than that of the blank test piece (mild steel). 


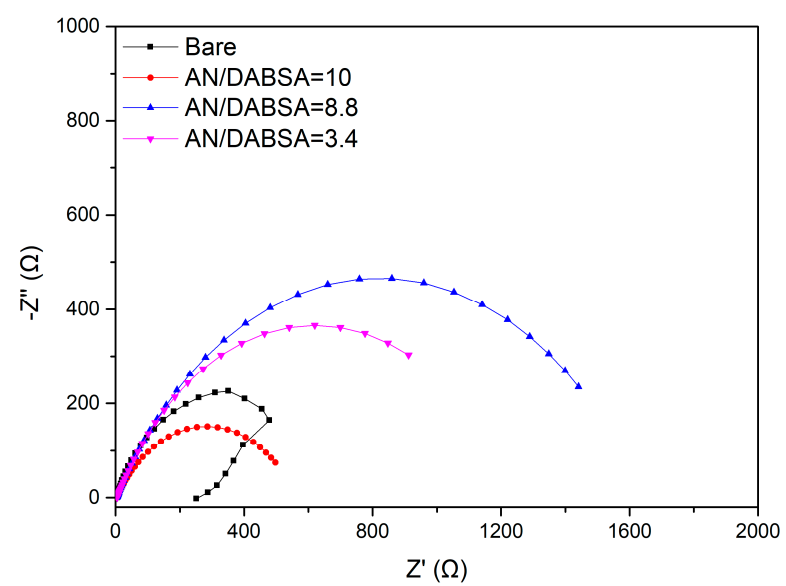

Figure 11. Nyquist plots of bare and SPAN-deposited mild steels with different AN/DABSA ratios in $1 \mathrm{M} \mathrm{NaCl}$.

Table 3. Charge transfer resistance $\left(R_{\mathrm{ct}}\right)$ derived from EIS measurements (Figure 11) and protection efficiency (PE) of bare and SPAN-deposited mild steels with different AN/DABSA ratios in $1 \mathrm{M} \mathrm{NaCl}$.

\begin{tabular}{ccc}
\hline Sample & $\boldsymbol{R}_{\mathbf{c t}}\left(\boldsymbol{\Omega} \mathbf{~ c m}^{\mathbf{2}}\right)$ & PE (\%) \\
\hline Bare & $246.5(2.01)$ & - \\
AN/DABSA $=10$ & $494.0(4.08)$ & $50.1(0.42)$ \\
AN/DABSA $=8.8$ & $1437.5(22.46)$ & $82.9(1.29)$ \\
AN/DABSA $=3.4$ & $906.9(17.10)$ & $72.9(1.37)$ \\
\hline
\end{tabular}

The data in parentheses represent the standard deviation.

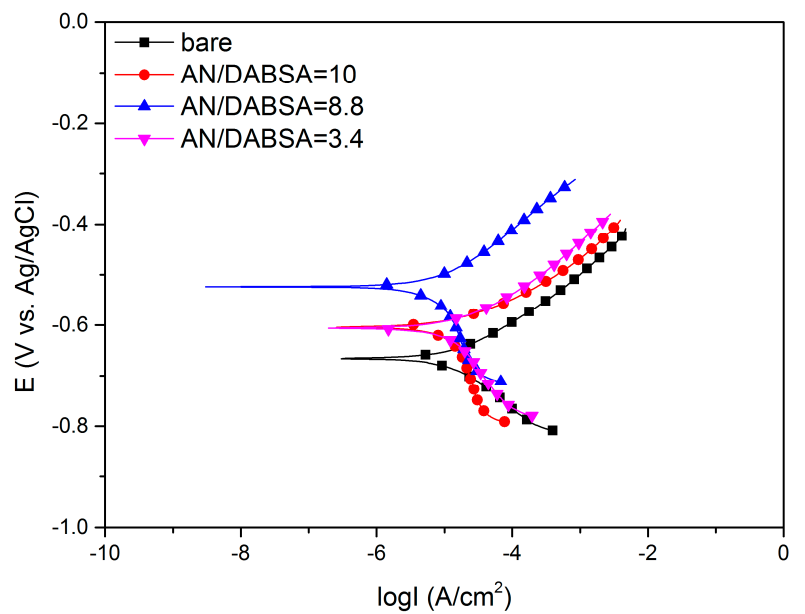

Figure 12. Polarization curves of bare and SPAN-deposited mild steels with different AN/DABSA ratios in $1 \mathrm{M} \mathrm{NaCl}$.

Table 4. Electrochemical parameters obtained by using polarization on bare and SPAN-deposited mild steels with different AN/DABSA ratios in $1 \mathrm{M} \mathrm{NaCl}$.

\begin{tabular}{ccccc}
\hline Sample & $\boldsymbol{E}_{\text {corr }}(\mathbf{m V}$ vs. $\mathbf{A g} / \mathbf{A g C l})$ & $\boldsymbol{I}_{\text {corr }}(\boldsymbol{\mu} \mathbf{A})$ & $\boldsymbol{R}_{\mathbf{p}}\left(\boldsymbol{\Omega} \mathbf{~ c m}^{\mathbf{2}}\right)$ & C.R. $\left(\mathbf{m m ~ y e a r}^{-\mathbf{1}}\right)$ \\
\hline Bare & $-665(13.04)$ & $20.9(0.41)$ & $1184.4(23.23)$ & $0.24(0.004)$ \\
AN/DABSA $=10$ & $-604(8.86)$ & $14.3(0.21)$ & $1946.1(28.57)$ & $0.16(0.002)$ \\
AN/DABSA $=8.8$ & $-523(11.88)$ & $8.8(0.20)$ & $4169.0(94.75)$ & $0.10(0.002)$ \\
AN/DABSA $=3.4$ & $-606(19.34)$ & $14.1(0.45)$ & $1916.1(61.15)$ & $0.16(0.005)$ \\
\hline
\end{tabular}


The reactions of carbon steel in $\mathrm{NaCl}$ solutions were as follows:

$$
\begin{gathered}
\mathrm{Fe} \rightarrow \mathrm{Fe}^{+2}+2 \mathrm{e}^{-} \\
\mathrm{Fe}^{+2} \rightarrow \mathrm{Fe}^{+3}+1 \mathrm{e}^{-} \\
\mathrm{O}_{2}(\mathrm{~g})+2 \mathrm{H}_{2} \mathrm{O}+4 \mathrm{e}^{-} \rightarrow 4 \mathrm{OH}^{-} \\
2 \mathrm{Fe}^{+2}(\mathrm{aq})+\mathrm{O}_{2}(\mathrm{~g})+2 \mathrm{H}_{2} \mathrm{O} \rightarrow 2 \mathrm{FeOOH}+2 \mathrm{H}^{+}
\end{gathered}
$$

The formation of a FeOOH layer provided protection for the metal and prevented it from corrosion. In a $1 \mathrm{M} \mathrm{NaCl}$ solution, SPAN (EB) was converted to SPAN (ES) via the oxygen reduction reaction.

$$
2 \mathrm{O}_{2}+4 \mathrm{H}_{2} \mathrm{O}+\mathrm{SPAN}(\mathrm{EB}) \rightarrow \mathrm{SPAN}(\mathrm{ES})+8 \mathrm{OH}^{-}
$$

As long as the polyaniline was in conducting (ES) form, it could provide protection to the metal and this still persisted with complete coverage in the coating. SPAN coating also works as a barrier against the diffusion of the electrolyte $(\mathrm{NaCl})$ and aggressive ions $\left(\mathrm{Cl}^{-}\right)$, protecting the substrate metal. In addition, the oxidation of metal can give rise to the reduction of SPAN (ES) to SPAN (EB) in the SPAN deposit film, leading to the anodic protection of a passive, protective $\mathrm{FeOOH}$ layer $[27,28]$. The mechanism set out in Scheme 2 can also be employed to explain the corrosion protection of SPAN on mild steel in $1 \mathrm{M} \mathrm{NaCl}$. One significant difference is that $\mathrm{Na}^{+}$cations in $\mathrm{NaCl}$ solution cannot dope SPAN as effectively as $\mathrm{H}^{+}$cations in $\mathrm{HCl}$ solution, resulting in an incomplete ES state for the SPAN. As a result, the relative efficiency of SPAN corrosion protection is lower in $\mathrm{NaCl}$ than in $\mathrm{HCl}$ (refer to Tables 1 and 3). The anti-corrosion ability of the SPAN film with an AN/DABSA ratio of 10 is lower than that of a SPAN film with an AN/DABSA ratio of 8.8, because the content of sulfonate anion in the former film is lower than that in the latter film. The low content of sulfonate anion is not effective in preventing the $\mathrm{Cl}^{-}$anion from attacking the metal substrate through the deposited polymer layer, resulting in the corrosion of the metal substrate. The SPAN film with an AN/DABSA ratio of 3.4 possessed the highest content of sulfonate anion and the highest hydrophilicity, the two effects of high sulfonate anions, namely blocking the attack of the $\mathrm{Cl}^{-}$anion and high hydrophilicity would offset each other. As a result, the SPAN film with AN/DABSA ratio of 8.8 has the optimal anti-corrosion.

\section{Conclusions}

We have presented the first demonstration of self-doped polyaniline films that incorporate 2,5-diaminobenzenesulfonic acid in aniline for corrosion protection coatings. The self-doped polyaniline films were obtained using electrodeposition by the direct electropolymerization of aniline and 2,5-diaminobenzenesulfonic acid on mild steel, without the addition of extra inorganic acid. The coatings exhibited effective protection of the surface of mild steel because of the significant prevention of $\mathrm{Cl}^{-}$and $\mathrm{O}_{2}$ attacks by forming an inert barrier. The optimal content of 2,5-diaminobenzenesulfonic acid in a polymer film, namely an aniline/2,5-diaminobenzenesulfonic acid ratio of 8.8, enhances the prevention of $\mathrm{Cl}^{-}$and $\mathrm{O}_{2}$ attack and is responsible for the highly desirable anticorrosion properties. As such, copolymers of aniline and 2,5-diaminobenzenesulfonic acid have excellent potential to be used as corrosion protection coating materials in a $\mathrm{HCl}$ or $\mathrm{NaCl}$ medium. As it convenient to obtain the anti-corrosion films using direct polymerization on a metal substrate without adding inorganic acid, we believe that electrodeposited self-doped polyaniline films will create a category of corrosion protection materials in the future and possibly replace other polymers.

Author Contributions: Jhen-Wei Wu performed the experiments; Chien-Hsin Yang, Wen-Churng Lin, and Tzong-Liu Wang conceived and designed the experiments; Jhen-Wei Wu, Hung-Yin Lin, Mei-Hwa Lee, and Chien-Hsin Yang analyzed the data; Hung-Yin Lin, Chien-Hsin Yang, and Tzong-Liu Wang contributed reagents/materials/analysis tools; Chien-Hsin Yang, Jhen-Wei Wu, Wen-Churng Lin, Hung-Yin Lin, Mei-Hwa Lee, and Tzong-Liu Wang wrote the paper. 
Acknowledgments: The authors greatly acknowledge financial support from the Ministry of Science and Technology in Taiwan (MOST 106-2221-E-390-025 and MOST 106-2622-E-390-001-CC2).

Conflicts of Interest: The authors declare no conflict of interest. The founding sponsors had no role in the design of the study; in the collection, analyses, or interpretation of data; in the writing of the manuscript, and in the decision to publish the results.

\section{References}

1. Santos, J.; Mattoso, L.; Motheo, A. Investigation of corrosion protection of steel by polyaniline films. Electrochim. Acta 1998, 43, 309-313. [CrossRef]

2. Cai, K.; Zuo, S.; Luo, S.; Yao, C.; Liu, W.; Ma, J.; Mao, H.; Li, Z. Preparation of polyaniline/graphene composites with excellent anti-corrosion properties and their application in waterborne polyurethane anticorrosive coatings. RSC Adv. 2016, 6, 95965-95972. [CrossRef]

3. Li, H.; Wang, X.; Zhang, L.; Hou, B. Preparation and photocathodic protection performance of CdSe/reduced graphene oxide/ $\mathrm{TiO}_{2}$ composite. Corros. Sci. 2015, 94, 342-349. [CrossRef]

4. Inagaki, M. Carbon coating for enhancing the functionalities of materials. Carbon 2012, 50, 3247-3266. [CrossRef]

5. Khan, M.I.; Chaudhry, A.U.; Hashim, S.; Zahoor, M.K.; Iqbal, M.Z. Recent developments in intrinsically conductive polymer coatings for corrosion protection. Chem. Eng. Res. Bull. 2010, 14, 73-86. [CrossRef]

6. Mooss, V.A.; Bhopale, A.A.; Deshpande, P.P.; Athawale, A.A. Graphene oxide-modified polyaniline pigment for epoxy based anti-corrosion coatings. Chem. Pap. 2017, 71, 1515-1528. [CrossRef]

7. Deshpande, P.P.; Jadhav, N.G.; Gelling, V.J.; Sazou, D. Conducting polymers for corrosion protection: A review. J. Coat. Technol. Res. 2014, 11, 473-494. [CrossRef]

8. Liao, Y.; Strong, V.; Chian, W.; Wang, X.; Li, X.-G.; Kaner, R.B. Sulfonated polyaniline nanostructures synthesized via rapid initiated copolymerization with controllable morphology, size, and electrical properties. Macromolecules 2012, 45, 1570-1579. [CrossRef]

9. Wessling, B. Passivation of metals by coating with polyaniline: Corrosion potential shift and morphological changes. Adv. Mater. 1994, 6, 226-228. [CrossRef]

10. DeBerry, D.W. Modification of the electrochemical and corrosion behavior of stainless steels with an electroactive coating. J. Electrochem. Soc. 1985, 132, 1022-1026. [CrossRef]

11. Wei, Y.; Wang, J.; Jia, X.; Yeh, J.M.; Spellane, P. Polyaniline as corrosion protection coatings on cold rolled steel. Polymer 1995, 36, 4535-4537. [CrossRef]

12. Williams, G.; Mamurray, H.N. Polyaniline inhibition of filiform corrosion on organic coated AA2024-T3. Electrochim. Acta 2009, 54, 4245-4252. [CrossRef]

13. Qiu, S.; Chen, C.; Zheng, W.; Li, W.; Zhao, H.; Wang, L. Long-term corrosion protection of mild steel by epoxy coating containing self-doped polyaniline nanofiber. Synth. Met. 2017, 229, 39-46. [CrossRef]

14. Syed, J.A.; Tang, S.; Lu, H.; Meng, X. Water-soluble polyaniline-polyacrylic acid composites as efficient corrosion inhibitors for 316SS. Ind. Eng. Chem. Res. 2015, 54, 2950-2959. [CrossRef]

15. Motheo, A.J.; Pantoja, M.F.; Venancio, E.C. Effect of monomer ratio in the electrochemical synthesis of poly(aniline-co-o-methoxyaniline). Solid State Ion. 2004, 171, 91-98. [CrossRef]

16. Li, X.G.; Huang, M.R.; Lu, Y.Q.; Zhu, M.F. Synthesis and properties of processible copolymer microparticles from chloroanilines and aniline. J. Mater. Chem. 2005, 15, 1343-1352.

17. Ates, M. A review on conducting polymer coatings for corrosion protection. J. Adhes. Sci. Technol. 2016, 30, 1510-1536. [CrossRef]

18. McCafferty, E. Validation of corrosion rates measured by the Tafel extrapolation method. Corros. Sci. 2005, 47, 3202-3215. [CrossRef]

19. Yang, C.H.; Wen, T.C. Polyaniline derivative with external and internal doping via electrochemical copolymerization of aniline and 2,5-diaminobenzenesulfonic acid on $\mathrm{IrO}_{2}$-coated titanium electrode. J. Electrochem. Soc. 1994, 141, 2624-2632. [CrossRef]

20. Ping, Z. In situ FTIR-attenuated total reflection spectroscopic investigations on the base-acid transitions of polyaniline. Base-acid transition in the emeraldine form of polyaniline. J. Chem. Soc. Faraday Trans. 1996, 92, 3063-3067. [CrossRef] 
21. Miroslava, T.; Šeděnková, I.; Tobolková, E.; Stejskal, J. FTIR spectroscopic and conductivity study of the thermal degradation of polyaniline films. Polym. Degrad. Stab. 2004, 86, 179-185.

22. Yüce, A.O.; Mert, B.D.; Kardaş, G. Electrochemical and quantum chemical studies of 2-amino-4-methyl-thiazole as corrosion inhibitor for mild steel in $\mathrm{HCl}$ solution. Corros. Sci. 2014, 83, 310-316. [CrossRef]

23. Grgur, B.; Elkais, A.; Gvozdenović, M.; Drmanić, S.; Trišović, T.L.; Jugović, B. Corrosion of mild steel with composite polyaniline coatings using different formulations. Prog. Org. Coat. 2015, 79, 17-24. [CrossRef]

24. Dkhireche, N.; Dahami, A.; Rochdi, A.; Hmimou, J.; Touir, R.; Touhami, M.E.; El Bakri, M.; El Hallaoui, A.; Anouar, A.; Takenouti, H. Corrosion and scale inhibition of low carbon steel in cooling water system by 2-propargyl-5-o-hydroxyphenyltetrazole. J. Ind. Eng. Chem. 2013, 19, 1996-2003. [CrossRef]

25. Danaee, I.; Khomami, M.N.; Attar, A. Corrosion behavior of AISI 4130 steel alloy in ethylene glycol-water mixture in presence of molybdate. Mater. Chem. Phys. 2012, 135, 658-667. [CrossRef]

26. Li, P.; Tan, T.; Lee, J. Corrosion protection of mild steel by electroactive polyaniline coatings. Synth. Met. 1997, 88, 237-242. [CrossRef]

27. Holness, R.J.; Williams, G.; Worsley, D.A.; McMurray, H.N. Polyaniline inhibition of corrosion-driven organic coating cathodic delamination on iron. J. Electrochem. Soc. 2005, 152, B73-B81. [CrossRef]

28. Williams, G.; Gabriel, A.; Cook, A.; McMurray, H.N. Dopant anion effects in the inhibition by polyaniline of corrosion-driven organic coating delamination on iron. J. Electrochem. Soc. 2006, 153, B425-B433. [CrossRef]

29. Sathiyanarayanan, S.; Muthukrishnan, S.; Venkatachari, G.; Trivedi, D.C. Corrosion protection of steel by polyaniline (PANI) pigmented paint coating. Prog. Org. Coat. 2005, 53, 297-301. [CrossRef]

30. De Souza, S.; da Silva, J.E.P.; de Torresi, S.I.C.; Temperini, M.L.A.; Torresi, R.M. Polyaniline based acrylic blends for iron corrosion protection. Electrochem. Solid-State Lett. 2001, 4, B27-B30. [CrossRef]

31. Kendig, M.; Hon, M.; Warren, L. Smart corrosion inhibiting coatings. Prog. Org. Coat. 2003, 47, 183-189. [CrossRef]

32. Sazou, D.; Kourouzidou, M. Electrochemical synthesis and anticorrosive properties of Nafion $^{\circledR}$ poly(aniline-co-aminophenol) coatings on stainless steel. Electrochim. Acta 2009, 54, 2425-2433. [CrossRef]

33. Kosseoglou, D.; Kokkinofta, R.; Sazou, D. FTIR spectroscopic characterization of Nafion ${ }^{\circledR}$-polyaniline composite films employed for the corrosion control of stainless steel. J. Solid State Electrochem. 2011, 15, 2619-2631. [CrossRef] 